\title{
ACUTE COMPLICATIONS OF MECKEL'S DIVERTICULUM
}

\author{
By H. Annamunthodo, F.R.C.S. \\ Senior Surgical Registrar, King George Hospital, Ilford
}

As the last review of this subject was in 1947 , a further discussion with illustrative cases is probably appropriate.

The incidence of Meckel's Diverticulum is I to 2 per cent. It is the commonest anomaly in the intestinal tract. H. Harkins (1933) found 327 in 25,149 routine necropsies ( 1.3 per cent.). H. Curd (1933) found 367 in 40,439 necropsies.

In $1947 \mathrm{~S}$. Taylor recorded I I cases of acute complications of Meckel's Diverticulum treated at King's College Hospital during 1928-1945. W. Atwood reported 37 cases out of 69,000 admissions. Of the 12 cases described here, six have occurred in King George Hospital during I95I-I952 (admission about 6,000 per year), three were patients of $\mathrm{Mr}$. Harold Dodd (cases I, 2, 4), notes and photographs of two cases of Littre's hernia (cases 7,8 ) have been supplied by Dr. J. Stasiak and one case Meckel's Diverticulum causing recurrent abdominal pain is included.

\section{Embryology}

Normally the vitelline duct loses its connection with the apex of the original mid gut loop before the latter is enclosed in the abdominal cavity. A Meckel's Diverticulum is the result of the incomplete obliteration of the vitelline duct. The diverticulm is usually 2 to 3 inches long and present 6 to 18 inches from the ileocaecal junction, but Handfield-Jones and Porritt state that it may be present 39 inches above the ileocaecal valve in the adult. Occasionally it is found even higher up.

Developmentally it is attached to the ileum at its antimesenteric border, but as a result of inflammation it may become fixed by adhesions to one or other side of the mesentery giving the appearance of attachment to the mesenteric border of the gut. This was seen in Case I $I$.

In the embryo, at the time when separation occurs, the vitelline duct possesses an epithelial lining which has the same developmental potentialities as the intestinal entoderm itself, hence it is not surprising to find heterotopic tissue in the diverticulum; 25 per cent. of diverticula show this anomaly (A. Taylor I94I). Indeed, a Meckel's Diverticulum may contain tissues of any part of 0 the alimentary tract and these may develop their characteristic pathological changes in their ectopic $\stackrel{\omega}{+}$ position. This explains the variability in the mode of onset of symptoms.

\section{Acute Symptoms}

These may be divided into: I. Obstructive, II. Inflammatory and III. Haemorrhagic.

The incidence of these symptoms is approximately the same. M. Rothman and D. Zetena (1943) and J. Haber (1947) found intestinal obstruction to be the commonest complicatiog of Of 1,605 cases in the literature, W. Moses (1947 or found 383 cases of intestinal obstruction whit haemorrhage was the presenting symptom in 496 . S. Taylor (1947) decribed one case of haemorrhage, six of intestinal obstruction and four of Meckel's Diverticulitis.

\section{Intestinal Obstruction}

Obstruction may be divided into simple, $\vec{\partial}$ strangulation, intussusception or obstruction in a hernial sac (Littre's Hernia).

\section{Simple Obstruction}

Often, at the site of the diverticulm or proximal to it, the ileum is narrowed. This may be due to 8 defective development of the segment of the ileum or constriction resulting from ulceration. 을 Indeed, occasionally this constriction is present in the terminal ileum in the absence of a diverticulum, in which case it is probably the result of an exag- $\bar{N}$ geration in the normal process of obliteration of $O$ the vitelline duct. When present, impaction of a $\tilde{O}$ foreign body may precipitate intestinal obstruction. N

\section{Strangulation}

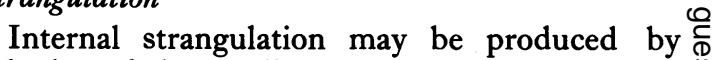
volvulus of the small gut around a diverticulum $\stackrel{\oplus}{+}$ with congenital attachment from its tip to the abdominal wall (a patent vitelline duct or a completely obliterated duct persisting as a fibrous 
band may act as the axis of a volvulus in the same way) or by a long diverticulum entwining a loop of gut (a free band from the apex of the diverticulum may do the same). Nevertheless, a distended diverticulum without any attachment to the abdominal wall may form the axis of the volvulus.

Of these 12 cases, three were intestinal strangulation. Two of these were due to a long diverticulum ensnaring a loop of gut and one to a free band from the apex of the diverticulum acting in the same way.

Case i. Male: Age 7.

History-Eight hours central abdominal pain and nausea; vomited once.

Examination-Temp. $99^{\circ}$, pulse 90 ; tenderness and guarding in R.I.F.

Diagnosis-Acute appendicitis.

At operation a long Meckel's Diverticulum was found ensnaring a congested loop of ileum. Gut viable. Diverticulectomy and appendicectomy were performed. Convalescence uneventful.

Case 2. Male: Age 6.

History-Twelve hours abdominal pain; vomited twice; increasing pulse rate.

Examination-Temp. $99^{\circ}$; tenderness and guarding in R.I.F.

Diagnosis-Acute appendicitis.

At operation a loop of ileum was found strangulated by a long Meckel's Diverticulum wrapped twice around the base. Fifteen inches of non viable bowel was resected and diverticulectomy was performed. Convalescence uneventful.

Case 3. Male: Age 38.

History -Sudden onset of acute abdominal pain; small vomits every $I-I \frac{1}{2}$ hours; no previous indigestion; B.O.R.

Examination-Temp. normal; pale; lies quietly; tongue furred; generalized abdominal tenderness and board-like rigidity most marked in upper quadrants; bowel sounds normal. P.R.tender in both I.F.

Diagnosis-Perforated peptic ulcer.

Conserative treatment for perforated peptic ulcer was instituted. Twelve hours later, abdominal pain which was relieved by original stomach aspiration recurred.

Examination-Temp. normal; pulse rate steady at 100; tenderness, guarding and rebound tenderness localized to R.I.F.

Diagnosis-Acute appendicitis.

At operation a loop of deeply congested small gut about six feet long was found strangulated by a free band from the tip of a Meckel's Diverticulum present at 18 inches from the ileocaecal valve. There was blood stained fluid in the peritoneal cavity. The appendix was normal. The gut appeared viable. One doubtful patch at the site of constriction was reinforced with free omental graft and diverticulectomy performed.

In convalescence he developed a pelvic abscess which was drained via the rectum, right subphrenic abscess which was drained and then signs of intestinal obstruction relieved by spontaneous faecal fistula.

Three months later, operation was performed for persistent faecal fistula. Sixteen inches of terminal ileum was resected and ileocaecal anastomosis performed. Has since been well.

In none of these cases was the correct diagnosis made preoperatively. Cases $I$ and 2 were regarded as acute appendicitis; in Case 3 a perforated peptic ulcer was suspected and treated but later developments suggested acute appendicitis.

\section{Intussusception}

W. Atwood (1946) described one case of intussusception in a series of 37 cases. H. Harkins (1933) found 160 cases in the literature and pointed out the following differences from the commoner intussusception of infants: (a) it occurred in older children; (b) there was generally a history of previous attacks of abdominal pain; (c) progress was milder and more chronic; (d) vomiting was usually intense.

Three of the 12 presented with intussusception.

\section{Case 4. Male: Age i 8 Months.}

History-Abdominal pain with passage of blood stained mucus per rectum.

Examination - Very ill child; palpable swelling left flank.

Diagnosis-Intussusception.

At operation, intussusception with Meckel's Diverticulum at the apex was reduced with difficulty and abdomen closed. Six weeks later, diverticulectomy was performed. Convalescence uneventful.

\section{Case 5. Male: Age 4.}

History-Three days abdominal pain and vomiting. B.O. daily.

Examination-Temp. 99.8; pulse 130 ; frail fretful child; tongue furred but moist; abdomen -no tenderness, guarding or distension, no palpable swelling, bowel sounds normal. P.R.vaguely tender high up, no palpable tumour; no blood on examining finger.

Observed for four days during which time his' pulse rate remained constant at I IO-120; vomited twice-small quantity of bile stained fluid, B.O.R. -formed stools with no blood; but there was slowly increasing abdominal distension. On the 


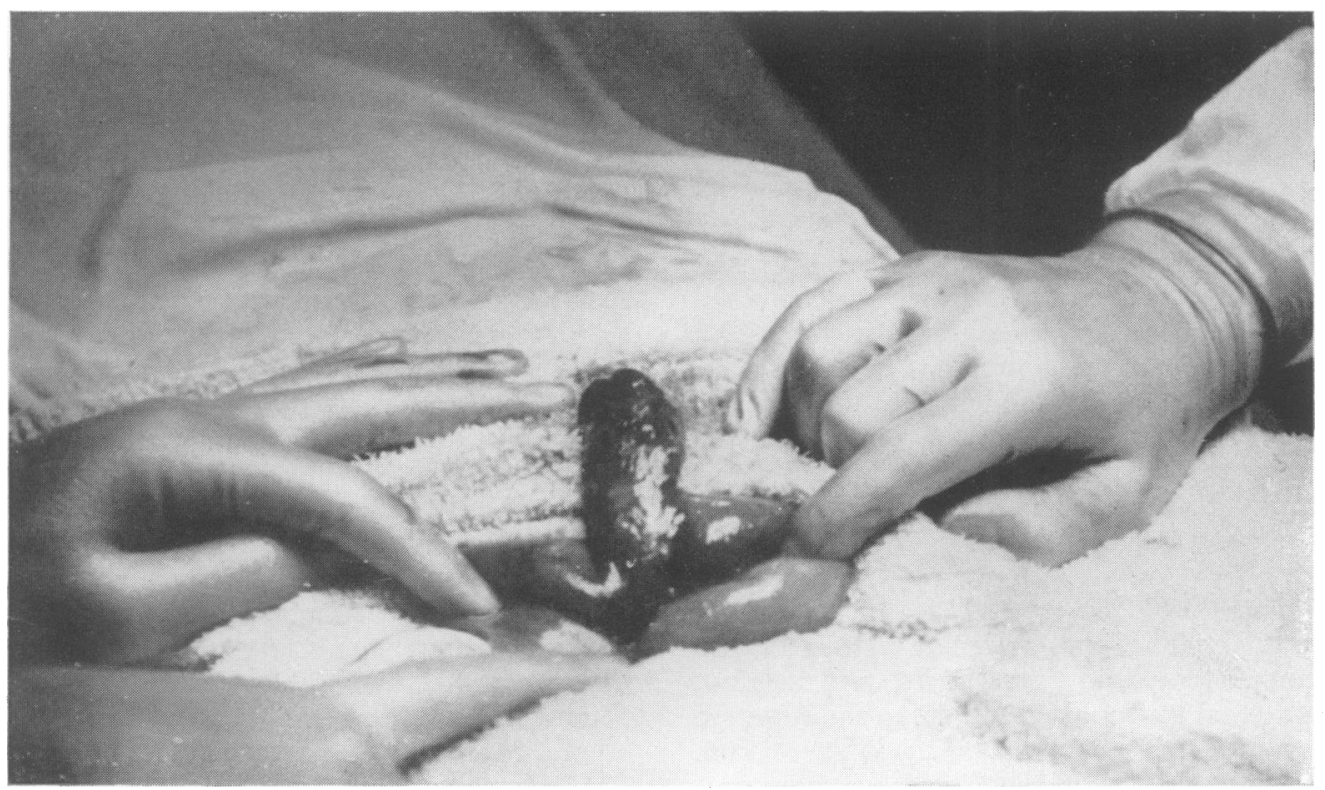

Case No. 8.

fourth day: not vomited for over 24 hours; no bowel action for 24 hours.

Examination-Temp. normal; pulse I Io; abdomen distended and tympanitic, no palpable swelling, bowel sounds increased. P.R.- no palpable swelling, no blood on finger.

Diagnosis-Intestinal obstruction probably due to intussusception.

At operation a gangrenous intussusception with a Meckel's Diverticulum at the apex was found. The outer coat of the intussusception perforated during reduction. Eight inches of ileum resected and end-to-end anastomosis performed. Convalescence uneventful.

\section{Case 6. Female: Age i2.}

History-6 $\frac{1}{2}$ hours central abdominal pain later localized to R.I.F.; vomited three times; four loose stools in six hours with no blood.

Examination-Temp. 99.4; pulse 80; tongue furred; tenderness in R.I.F.; Rovsing's sign negative; bowel sounds normal. P.R.-Tender R.I.F.; no blood on examining finger.

At operation, intussusception with Meckel's Diverticulum forming the apex was reduced. Diverticulectomy and appendicectomy were performed. Convalescence uneventful.

These patients were easier to assess than the cases of intestinal strangulation. They, were in the older age group varying from 18 months to 12 years as opposed to the typical intussusception in infants which usually occur from 4 to 12 mont There was no history of previous abdominal pan in any of them. Case 4 was critically ill admission. Case 5 was less anxious; indeed, days passed before the diagnosis was made. In Case 6 the symptoms were mild and more sug-气̃ gestive of appendicitis. Vomiting was not å prominent feature. In Case 4 blood and mucus $\vec{B}$ were passed per rectum but not in the other two.3

\section{Littre's Hernia}

Of 1,605 cases in the literature, W. Moses (1947) found 188 (II.7 per cent.) presented with a?. strangulated hernia (Littre's Hernia). Theo clinical picture is illustrated by the following two cases.

Case 7. Female: Age 79.

History -2 days generalized abdominal pain and vomiting. Patient in confused state and unable to을. give a complete history; evidently does notr realize that there is a tender lump in left groin.? Relatives state that lump was present for 2 months $\tilde{O}$ with intermittent attacks of central abdominal pain.

Diagnosis-Strangulated left femoral hernia.O Operation was performed under local anaeseo thetic because of poor condition of patient. Femoral hernial sac was found containing.? gangrenous Meckel's Diverticulum. Adjacentए knuckle of ileum also gangrenous. Resection was $\frac{\text { ? }}{\mathrm{P}}$ performed and continuity of the bowel restored 
end-to-end anastomosis. Lotheisen's herniorrhaphy performed. Convalescence uneventful.

Case 8. Female: Age 56.

History-6 hours central abdominal pain with tender swelling in right groin. Nausea but no vomiting.

Diagnosis-Strangulated right femoral hernia.

At operation the femoral hernial sac contained congested Meckel's Diverticulum 4 in. long with adhesions between the bowel and the sac. The diverticulum was resected and Lotheisen's herniorraphy performed.

On seventh day the patient developed a penicillin rash with pyrexia and died on the thirteenth day of massive pulmonary embolus.

\section{Inflammation}

Once it is realized that a Meckel's Diverticulum may contain lymphoid tissue in its wall, it is not surprising that it should be subject to the common disorder of the appendix i.e., inflammation. Normally the Meckel's Diverticulum communicates with the intestine through a wide opening, the wall is muscular and the contents liquid so that it empties easily. Hence, diverticulitis is rare. It is more likely to occur in a diverticulum with a narrow neck and may be precipitated by impaction of a foreign body. Diagnosis of appendicitis is usually made.

Case 9. Female: Age 8.

History - 5 days abdominal pain localized to R.I.F.; nausea but no vomiting; pain worse for 24 hours; no bowel action for 2 days.

Examination-Temp. 100; pulse 100; tongue furred; tenderness, guarding and rebound tenderness in R.I.F. P.R.-tender R.I.F.

Diagnosis-Acute appendicitis, with peritonitis.

At operation a gangrenous perforated Meckel's Diverticulum was found. The neck of the diverticulum was constricted to the size of the normal appendicular lumen. Diverticulectomy and appendicectomy were performed. Convalescence uneventful.

\section{Haemorrhage}

As stated, any of the tissues of the alimentary tract may be sequestered in the wall of a Meckel's Diverticulum.

V. Hunt and H. Bonesteel (1934) found I3 recorded cases of aberrent pancreatic tissue in the wall of the diverticulum but no proved case of pancreatitis. H. Hudson and L. Koplik (1932) reported the presence of colonic mucosa.

The common heterotopic tissue to give rise to symptom is gastric mucosa. This occurs in 15 to 20 per cent. of diverticula (R. Greenblatt et al,
1936). It may line the tip or even the whole of the diverticulum. When peptic ulceration occurs it is usually present not in the aberrent tissue but in the adjacent intestinal mucosa resembling the stomal ulcer following gastro-jejunostomy. Eighty per cent. of these ulcers occur in the male with a greater incidence in infancy and childhood (A. Taylor I94I). J. Matt and P. Timpone (1940) state that over 50 per cent. occur in the first decade and 75 per cent. before the age of $16 . \quad J$. Marshall (I942) reviewing 12 cases of diseased diverticula concluded that ulceration was the commonest complication in the younger patient while the older ones presented with intestinal obstruction or inflammation.

A peptic ulcer in a Meckel's Diverticulum is liable to the same complications as the gastric or duodenal ulcer. Cases of perforation have been reported, but the commonest complication is haemorrhage. J. Chesterman (1935) reported the first cases in Great Britain.

W. Moses (I947) finds 496 cases of haemorrhage of $\mathrm{I}, 605$ recorded cases of diseased diverticula (30.9 per cent.). S. Taylor (1947) described one case and mentioned two others. J. Walton and N. Lill (1952) described one case and stated that the cardinal symptom was haemorrhage, often copious, and usually intermittent, occurring a intervals of weeks or months.

A. Murray (1947) stressed that malaena from a Meckel's Diverticulum was intermediate in colour between the tarry stool of gastric and duodenal haemorrhage and the bright red bleeding from colonic haemorrhage. This depends on the severity of the haemorrhage as illustrated by the following case.

Case io. Male: Age $4 \frac{1}{2}$.

History - 4 days central abdominal pain followed by passage of bright red blood in the stool; since then the motions have been loose and contained altered blood in diminishing quantity.

Examination-Temp. 100.8; pulse 138 ; very pale; abdomen soft, non-tender with no palpable mass and normal bowel sounds. P.R.-bright red blood on examining finger.

Investigations- $\mathrm{Hb} . \quad 42$ per cent., R.B.C 2,200,000. Barium enema-normal. Barium swallow-' Terminal ileum appears to be somewhat irregular in outline and there is some suggestion of dilatation of the ileum proximal to it. The appearances are suggestive of terminal ileitis. No Meckel's Diverticulum demonstrated.' Sigmoidoscopy-normal up to $20 \mathrm{~cm}$.

Diagnosis-Haemorrhage from Meckel's Diverticulum.

Haemorrhage stopped and patient was discharged to return in 2 weeks for laparotomy. 
Returned in 5 days with further severe haemorrhage. Hb. 25 per cent.

After transfusion of I pint of blood laparotomy was performed. Four inches of oedematous ileum with Meckel's Diverticulm was resected and end-to-end anastomosis performed. Convalescence uneventful.

Pathologist's Report: 'Oedematous Meckel's Diverticulum with narrow neck. At the neck was a peptic ulcer typical in appearance $1 \mathrm{~cm} . \times \frac{1}{2} \mathrm{~cm}$. extending into the wall of the adjacent ileum. Pin point open vessel can be seen in the base. The wall of the diverticulum was lined with gastric mucosa.'

While haemorrhage from Meckel's Diverticulum is usually due to peptic ulceration, it may occur from other causes. S. Taylor (1947) described one case of haemorrhage from ulcerated leiomyoma. Other tumours-lipoma, myxoma, adenoma, carcinoid, carcinoma, leiomyosarcoma, spindle cell sarcoma - have been reported. Many of these presented with haemorrhage.

J. Walton and N. Lill (1952) recorded one case of haemorrhage in the absence of ulceration or tumour. The following case was similar.

\section{Case i i. Male: Age 64.}

History-Was in hospital for 4 weeks for course of physiotherapy for severe rheumatoid arthritis when he passed large quantities of bright red blood per rectum.

Examination-Pale; pulse good volume; abdomen not distended, no tenderness, no palpable mass. P.R.-bright red blood on examining finger.

In 24 hours he passed three large malaena stools consisting of blood clots and bright red blood. Increasing pallor, rising pulse rate with fall in B.P. to $60 / 40$. No blood aspirated from stomach by Ryle's tube. Much improved on transfusion of 6 pints of blood.

He continued to pass malaena stools for 4 days but no further bright red loss. On fifth day further passage of bright red blood and clots. Pulse rate rising and fall in B.P. Improved on transfusion of 2 pints of blood.

Diagnosis-Haemorrhage from small gut, probably Meckel's Diverticulum.

At operation, oedematous Meckel's Diverticulum adherent to the mesentery was resected with about 3 in. of adjacent ileum and end-to-end anastomosis performed. Other abdominal viscera were normal. Two pints of blood were transfused during the operation and in the immediate post-operative period. Convalescence uneventful. No further haemorrhage up to 9 months later.

Pathologist's Report: 'Diverticulum 2 in. long, narrow at neck. Mucosa shows polymorphonuclear cells infiltration but no actual ulcerative lesion. ڤ్ No heterotopic tissue found.'

N.B.-No anti-rheumatic drug was admini-免 stered.

It is inconceivable that haemorrhage of this $\Rightarrow$ severity necessitating massive transfusion ands? emergency operation should occur in the absence of a breach in the mucosa. When ulceration occurs in a Meckel's Diverticulum it is often $\frac{\bar{s}}{\neg}$ present not in the heterotopic gastric mucosa but in the adjacent ileum and one explanation of the haemorrhage in this case is an acute ulcer either atthe site of resection or in the adjacent ileum. This will heal once the acid secreting gastric $\vec{\omega}$ mucosa in the diverticulum is removed. In this specimen, however, no heterotopic gastric mucosa을 was found to complete the explanation. However, J. Chesterman (1935) pointed out that ulceration could occur in the absence of gastric mucosa.w Irregular peristalsis producing invagination of the apex of the diverticulum has been suggested as the $\overrightarrow{0}$ cause in these cases.

\section{Other Complications}

S. Taylor (1947) described one case of foreign body impaction. A sharp foreign body e.g., fish bone, may perforate the diverticulum and $S \cdot \overrightarrow{.}$ Linquist (1926) recorded a case of perforation gyo fragment of wood.

I. MacDonald (1947) collected nine cases tuberculous infection of Meckel's Diverticulưm and recorded one case which was secondary to a⿳亠二口犬 pulmonary lesion but with no sign of tuberculosiső elsewhere in the intestinal tract.

\section{Chronic Symptoms}

S. Taylor (1947) found no cases of chronic or? subacute symptoms due to Meckel's Diverticulum: in the literature. He described one of recurrent abdominal pain cured by removal of the offending diverticulum and stated: "This case suggests the 3 possibility of such a condition being found if it is kept in mind and provides another good reason for윽 looking for Meckel's Diverticulum after every appendicectomy. It is precisely in those patients 근 with a not too clear clinical picture of appendicitis $?$ that the condition is most likely to be encountered.'

In the following case, laparotomy was performed $N_{\sigma}^{N}$ for recurrent attacks of abdominal pain diagnosed as appendicitis.

Case 12. Female: Agé 33.

History-Recurrent attacks of pain in R.I.F. for $\stackrel{0}{\frac{0}{D}}$ I6 years, each attack lasting a few days; attacks $\stackrel{\oplus}{?}$ more frequent and more severe for 4 years; 0 B.O.R.; no frequency or dysuria; no dysmenorrhoea. 
Examination-Healthy woman; tender in R.I.F. on deep pressure. P.R.-tender in R.I.F.

At operation the appendix was normal. A Meckel's Diverticulum was present. The neck of the diverticulum was narrow and the lumen was constricted in the middle with distension of the apex beyond this point. Other abdominal viscera were normal. Diverticulectomy and appendicectomy were performed. Convalescence uneventful. Free of symptoms 6 months later.

Pathologist's Report: ' Inflammatory changes in Meckel's Diverticulum. No heterotopic tissue.'

\section{Discussion}

The diagnosis of Meckel's Diverticulum is rarely made pre-operatively and then it can be only by a process of exclusion. R. Harbin (1926) reported one correct pre-operative diagnosis in I3 cases and J. Harber (1947) one in 23. The diverticulum can be demonstrated radiologically but rarely (J. Rousseau and A. Martin 1943).

In intussusception occurring in older children a Meckel's Diverticulum is a real possibility but in other cases of intestinal obstruction the diagnosis is difficult. Indeed, in all three cases operation was performed on diagnosis of appendicitis. In any small gut obstruction, in the absence of any obvious cause, Meckel's Diverticulum must be considered as a possible aetilogical factor.

Meckel's Diverticulitis is indistinguishable clinically from appendicitis.

Meckel's Diverticulum is probably the commonest cause of small gut haemorrhage especially in children and has been recorded in an I I month old infant (R. Wilkinson I947). In any infant with painless rectal haemorrhage of undetermined origin a presumptive diagnosis of Meckel's Diverticulum may be made. Haemorrhage may be of severe degree necessitating massive transfusion and emergency operation. Ulcerating tumours of the small intestine may present the identical clinical picture.

\section{Conclusion}

This vestigial organ is a potential danger to life and should be looked for in every abdominal operation; when encountered it should be removed. The wisdom of this advice has been more than confirmed by the frequency and severity of the complications which may arise. Excision of the diverticulum is a simple procedure and the added risk of its removal in an abdominal operation is infinitesimal.

I am indebted to Mr. Harold Dodd, Mr. Hermon Taylor and Mr. C. P. Allen for permission to publish the case reports and for their help, encouragement and criticisms. I wish to thank Dr. J. Stasiak for the notes and photograph of the two cases of Littre's Hernia.

\section{BIBLIOGRAPHY}

ATWOOD, W. G. (1946), Nerw Eng. F. Med., 234, 329.

CHESTERMAN, J. T. (1935), B.Y.S., 23, 267.

CURD, H. H. (1933), Arch. Surg., 98, 1070.

GREENBLATT, R. B., et al. (1936), A.F.S., 3I, 285.

HARBER, J. J. (1947), A. ..S., 73, 468.

HARBIN, R. M. (1926), S.G.O., 42, 515.

HARKINS, H. N. (1933), Ann. Surg., 98, 1070.

HUDSON, H. W., and KOPLIK, L. H. (1932), New Eng. F. Med. 206, 827.

HUNT, V. C., and BONESTEEL, H. T. S. (1934), Arch. Surg. Chicago, 28, 425.

LINQVIST, S. (1926), Zbl. Chir., 53, 1756.

MacDONALD, I. B. (1947), B.F.S., 34, 324.

MARSHALL, J. F. (1942), N. Carolina M. F., 3, 192.

MATT, J. G., and TIMPONE, P. J. (I940), A.f.S., 47, 612.

MOSES, W. R. (1947), New Eng. F. Med., 237, I 18.

MURRAY, A. G. (1947), Med. F. Aust., 1, 145.

ROTHMAN, M., and ZETENA, D. F. (1943), A.7.S., 60, 443.

ROUSSEAU, J. P., and MARTIN, A. G. M. (1943), Radiology, 40, 605 .

TAYLOR, A. L. (1931), Bristol Med-Chir. f., 48, 113.

TAYLOR, S. (I947), Lancet ii, 786.

WALTON, J. N., and LILL, N. D. (1952), Brit. med. f., i, 88.

WILKINSON, R. W. (r947), B.F.S., 35, 285.
NOTICE OF SPECIAL INTEREST TO SUBSCRIBERS:

" WHY NOT HAVE YOUR COPIES OF THIS JOURNAL BOUND INTO YEARLY VOLUMES?"

\begin{abstract}
Arrangements have now been made to have the twelve monthly issues fully bound in dark green pin head cloth, lettered in gilt on spine with name of Journal, Volume Number and year, complete with index at front, 17s. 6d. per Volume, post free. A limited number of out of print journals are available to bind into volumes and make your library complete. Price on application giving details of issues required to complete back volumes.
\end{abstract}

MEDICINE

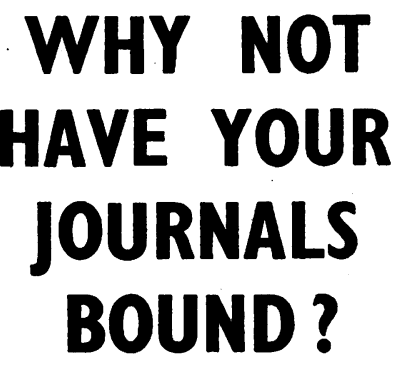

WHY NOT

HAVE YOUR

JOURNALS

BOUND? 